\title{
Luminescence properties of $\mathrm{LiGaO}_{2}$ crystal
}

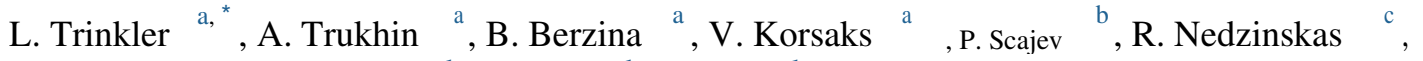 \\ S. Tumenas ${ }^{\text {c }}$, M.M.C. Chou ${ }^{\text {d }}$, L. Chang ${ }^{\text {d }}$, C.-A. Li ${ }^{\text {d }}$ \\ ${ }^{a}$ Institute of Solid State Physics, University of Latvia, Latvia \\ b Institute of Applied Research, Vilnius University, Lithuania \\ ${ }^{c}$ Center for Physical Sciences and Technology, Lithuania \\ ${ }^{\mathrm{d}}$ Department of Materials and Optoelectronic Science, National Sun Yat-Sen University, Taiwan
}

\begin{abstract}
a bs tract
The comprehensive spectral study of lithium metagallate $\mathrm{LiGaO}_{2}$ crystal has been done including methods of pump-probe techniques, optical absorption, photoluminescence, luminescence kinetics, thermoluminescence and polarised luminescence in broad temperature region. Luminescence spectrum of the crystal contains the main emission bands at 4.43, 3.76, 2.38 and 1.77 $\mathrm{eV}$. The novel data on luminescence excitation spectra including VUV area, kinetics and polarization are presented. The correlation between pump-probe experiment results and luminescence properties is found. Conclusions are done about the recombination character of all the observed emission bands, implying tunnel recombi-nation of donor-acceptor pairs.
\end{abstract}

\section{Introduction}

Lithium metagallate $\mathrm{LiGaO}_{2}$ is arousing interest of investigators as a material potentially attractive for various practical applications. Previously this crystal was studied due to its piezoelectric proper-ties [1] and proposed for ultrasonic devices [2], it was investigated as a laser material when doped with chromium [3] and vanadium ions [4]. Recently $\mathrm{LiGaO}_{2}$ was proposed as a lattice-matched sub-strate for heteroepitaxy of $\mathrm{GaN}$ [5], $\mathrm{InN}$ [6] and $\mathrm{ZnO}$ [7e9]. Alloying of $\mathrm{LiGaO}_{2}$ with $\mathrm{ZnO}$ creates a material with a band gap tunable in 3.3e5.6 eV range [10]. Besides when doped with cupper ions this crystal was studied as a material for optically and thermally stim-ulated dosimetry [11].

Lithium metagallate is a wide band gap ternary mixed-metal oxide compound (I-III-VI). $\mathrm{LiGaO}_{2}$ has a wurtzite-derived struc-ture with a particular ordering of $\mathrm{Li}$ and $\mathrm{Ga}$ cations accompanied by structural relaxation compensating slight difference in $\mathrm{LieO}$ and $\mathrm{GaeO}$ bonds [12]. The space group of $\mathrm{LiGaO}_{2}$ is Pna21 determining polarity of the crystal along c-axis [13]. $\mathrm{LiGaO}_{2}$ is optically negative biaxial crystal with a principal optical axis directed along c. Data on its band gap Eg varies from $5.26 \mathrm{eV}$ [14] to $5.76 \mathrm{eV}$ [15], but the most usual value referred at room temperature is $5.6 \mathrm{eV}$ [16]. In the recent paper [17] it was shown that the band gap is about $6.1 \mathrm{eV}$ and fundamental absorption edge starts with exciton transitions. The exciton energy, which corresponds to the band gap energy minus the exciton binding energy, was determined as 6.03, 6.08, and $5.92 \mathrm{eV}$ for Ejja, Ejjb, and Ejjc polarizations, respectively.

Though many potential applications of $\mathrm{LiGaO}_{2}$ imply use of its optical properties there are not many studies of its luminescence characteristics. As to our knowledge, there are some works devoted to luminescence of dopants in $\mathrm{LiGaO}_{2}$, such as ions of $\mathrm{Cr}^{3} \mathrm{p}_{[18,19], \mathrm{Cu}^{\mathrm{p}}}[11], \mathrm{v}^{3} \mathrm{p}_{\text {[4], but very few studies }}$ deal with luminescence of pure crystals e among them the early work of Dirksen [15], where the observed emission band at $360 \mathrm{~nm}$ and its excitation band at $220 \mathrm{~nm}$ were explained by charge transfer transitions between $\mathrm{Ga}$ and $\mathrm{O}$ ions, and the recent work [20] on luminescence of $\mathrm{LiGaO}_{2}$ nanoflakes with the same interpretation of the emission and excitation bands, located at lower energies compared to bulk crystal.

Recently a large $\mathrm{b}-\mathrm{LiGaO}_{2}$ single crystal was grown by a Taiwan group with an aim to provide a high quality lattice-matched sub-strate for $\mathrm{ZnO}$ heteroepitaxy [14]. The authors have done the basic characterization of the grown material including cath-odoluminesence at room temperature.
However, still more detailed study of $\mathrm{LiGaO}_{2}$ properties, especially those connected with lumi-nescence seems to be necessary. 
The aim of the present paper is investigation of the luminescent properties of pure lithium metagallate in general, and elucidation of luminescence mechanisms in particular.

\section{Materials and methods}

\subsection{Sample}

A large $\mathrm{b}-\mathrm{LiGaO}_{2}$ crystal was produced by Taiwan authors of this paper using Czochralski method from mixture of raw material powders of $\mathrm{LiCO}_{3}$ and $\mathrm{Ga}_{2} \mathrm{O}_{3}$ (5 $\mathrm{N}$ purity) according to stoichio-metric ratio [14]. The producers found that $\mathrm{LiGaO}_{2}$ evaporated congruently at the melting point in the used growth atmosphere, so neither Li nor Ga compensation was necessary. The bulk crystal was cut into samples along the main crystallographic directions.

Most of the experiments were done using the $\mathrm{LiGaO}_{2}$ (100) sample, having size of $10101 \mathrm{~mm}$. X-ray fluorescence elemental analysis showed presence of no impurities except of $\mathrm{Al}$ ions with concen-tration $0.2 \mathrm{w} \%$.

\subsection{Experimental}

$\mathrm{LiGaO}_{2}$ crystal was investigated using several luminescence and pumpprobe experimental methods for study of luminescence processes and nonequilibrium states of excited charge carriers.

Optical absorption was measured using a spectrophotometer (Specord 210 , Analytic Jena) in the $190 \mathrm{e} 1100 \mathrm{~nm}(6.5 \mathrm{e} 1.12 \mathrm{eV})$ spectral range. Study of thermal dependence of absorption was done inserting a sample into a closed cycle refrigerator (CCS-100/ 204, Janis Research Corporation), which provided fixed tempera-tures in the $10 \mathrm{e} 300 \mathrm{~K}$ range. For this experiment a sample with two polished faces was used.

Photoluminescence PL and photoluminescence excitation PLE were studied using two types of self-made experimental set-ups operating in UV-VIS and VUV ranges. 1. In the UV-VIS set-up excitation light in the $190 \mathrm{e} 400 \mathrm{~nm}(6.5 \mathrm{e} 3.1 \mathrm{eV})$ range was pro-vided either by a deuterium lamp (LDD-400) conjugated with a grating monochromator (MDR-2, LOMO) or a solid state laser DTL-389QT ( $263 \mathrm{~nm}$, or $4.71 \mathrm{eV})$; luminescence registration in $240 \mathrm{e} 900 \mathrm{~nm}(5.16 \mathrm{e} 1.38 \mathrm{eV})$ range was done either by photo-multipliers (Hamamatsu H7468-03, H7468-20) connected with a prism monochromator (SPM-2, Carl Zeiss) or with CCD camera (Andor DV 420A-BU2) conjugated with a grating monochromator (Andor Shamrock SR-303i-B); a sample was mounted on the cold finger of a close cycle refrigerator (CCS-100/204, Janis Research Corporation).

2. In the VUV set-up excitation sources were presented by $\operatorname{ArF}(193 \mathrm{~nm}$, or $6.42 \mathrm{eV}$, pulse energy of about $5 \mathrm{~mJ}$ with a duration of $5 \mathrm{~ns})$ and $\mathrm{F}_{2}(157$ $\mathrm{nm}$, or $7.90 \mathrm{eV}$, pulse energy of about $0.5 \mathrm{~mJ}$ with a duration of $5 \mathrm{~ns}$ ) pulse lasers (PSX-100, Neweks) and by a deute-rium discharge lamp with a $\mathrm{MgF}_{2}$ window (VUV Light Source Unit L10366 Series, Hamamatsu) conjugated with a $0.5 \mathrm{~m}$ vacuum monochromator (Seya-Namioka); luminescence detection was realized with a grating monochromator (MCD-2 Belorussia) and a photomultiplier tube (H6780-04, Hamamatsu). Sample tempera-ture in the $80 \mathrm{e} 300 \mathrm{~K}$ was provided by a liquid nitrogen cryostat, additional cooling down to $60 \mathrm{~K}$ was done by evaporation of liquid nitrogen. In both set-ups laser intensity was attenuated by means of metal sieve filters. All necessary instrumental spectral corrections were made.

Time-resolved photoluminescence (TRPL) measurements including time-resolved spectra and luminescence decay curves were performed on the VUV set-up, for luminescence detection using a photomultiplier tube H678004 (0.7 ns rise) with $50 \mathrm{Ohm}$ resistive load in time range $10^{9}$ e $10^{4} \mathrm{~s}$ and Hamamatsu H8259-
02 module in photon counting mode in time range $10^{5}$ e $10^{2}$ s. An oscilloscope (Textronic TDS 2022B) was exploited for decay curve registration. Each curve was averaged for 128 pulses. The time resolved PL spectra were measured by registration of the decay curve for each point of the PL spectrum in nanosecond and microsecond ranges.

Pump-probe measurements including Light induced transient grating (LITG) [21] and Differential transmittivity (DT) [22] were used to obtain information on ultra-fast carrier recombination phenomena during crystal excitation process. In DT experiments $\mathrm{LiGaO}_{2}$ sample was hit by a pump pulse, which generated excitation in the sample, then the sample was irradiated by a probe pulse and its transmittivity was measured. Analysis of DT gives information on the decay of the generated excitation, i.e. excited carriers. These measurements were done using picosecond Nd:YAG laser pulses at $213 \mathrm{~nm}(5.82 \mathrm{eV})$ (pump) for excitation and $1064 \mathrm{~nm}(1.17 \mathrm{eV})$ (probe) wavelengths for probing of excited carrier dynamics. Dif-ferential transmittivity DT $1 / 4 \mathrm{DT} / \mathrm{T}$, where $\mathrm{T}$ is the sample trans-mittivity for probe pulse at $1064 \mathrm{~nm}$ wavelength, determines absorption change, and thus excess carrier density in the sample. Detailed description of the equipment can be found in Ref. [23].

LITG decay was monitored by diffraction of a delayed probe beam at $1064 \mathrm{~nm}$ on interference pattern, generating a dynamic spatial diffraction grating in the sample and leading to refractive index modulation by free carriers and thus providing an instanta-neous diffraction efficiency $\mathrm{DE}(\mathrm{t})$.

Comparison of the LITG grating decay times, $t_{G}$, at few different grating periods $(\mathrm{L} 1 / 4$ 2, $4 \mathrm{~mm}$ ) al-lows determination of the carrier diffusion coefficient $\mathrm{D}$ and their lifetime tR as $1 / \mathrm{tg}_{\mathrm{G}} 1 / 41 / \mathrm{t}_{\mathrm{Rp}} 4 \mathrm{p}^{2} \mathrm{D} / \mathrm{L}^{2}$.

Thermoluminescence was measured on the UV-VIS experi-mental setup, using monochromator-filtered emission of the deuterium lamp for preliminary irradiation of the sample with definite wavelengths. Heating in the $10 \mathrm{e} 300 \mathrm{~K}$ temperature range was done with heating rate $2 \mathrm{~K} / \mathrm{s}$ in the helium closed cycle refrigerator.

Polarised luminescence was studied on the UV-VIS experi-mental setup, modified by insertion of polariser P1 (Rochon prism) in the excitation channel, and polariser P2 (UV polarization film) in the perpendicular luminescence detection channel. Besides, a depolariser was mounted before monochromator of the registra-tion system in order to prevent interference of polarised light with optical system of monochromator. During luminescence polariza-tion measurements P1 was oriented vertically, corresponding to vertical orientation of electric field vector of excitation light wave, and luminescence signal was detected for two orientations of P2 e vertical and perpendicular, denoted, correspondingly $\mathrm{I}_{0}$ and $\mathrm{I}_{90}$. Polarization degree was calculated according to a formula:

\section{Results}

\subsection{Optical absorption}

Investigation of spectral properties of $\mathrm{LiGaO}_{2}$ started with measurement of absorption spectra in $10 \mathrm{e} 300 \mathrm{~K}$ temperature range, shown in Fig. 1. The sample orientation was not determined during the absorption and luminescence measurements, so the results are averaged for all crystal polarizations. The curves are presented by the optical edge of fundamental absorption around $5.17 \mathrm{eV}$ at RT and a long absorption tail (shown up to 4 $\mathrm{eV}$ ) stretching up to $1 \mathrm{eV}$ without any features. The saturation level of optical density at value 3 is instrumental. Temperature rise from 10 


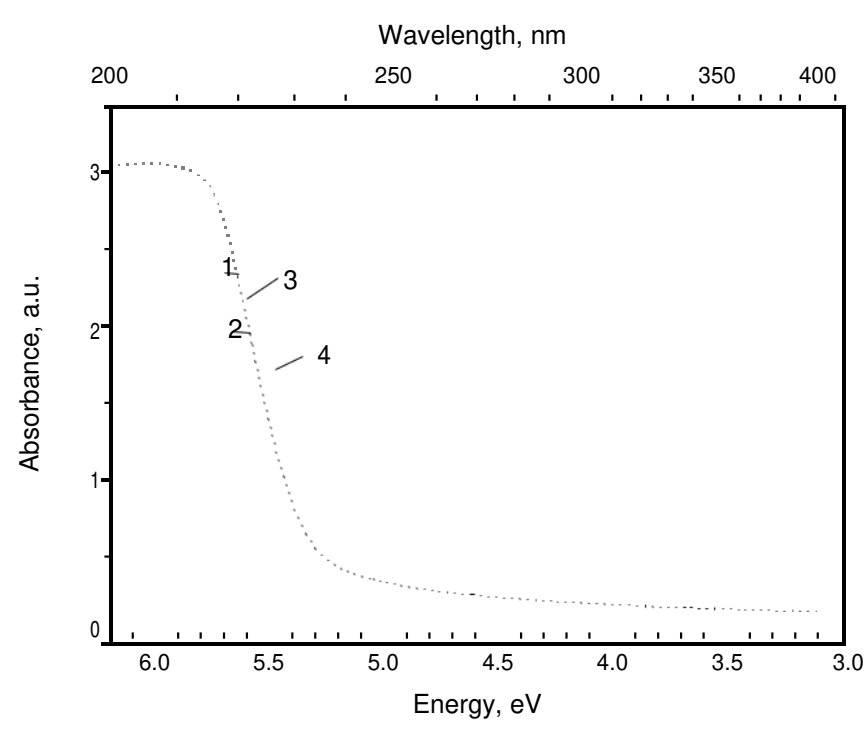

Fig. 1. Absorption spectra of $\mathrm{LiGaO}_{2}$ measured at $10 \mathrm{~K}(1), 100 \mathrm{~K}(2), 200$ (3) and $300 \mathrm{~K}$ (4).

to $300 \mathrm{~K}$ (curves 1 to 4 ) causes red shift of the optical band gap by $\sim 0.1 \mathrm{eV}$. The experimentally observed absorption edge for the sample investigated is located at lower energy than the value of the exciton energy, determined in Ref. [17] (5.92e6.03 eV, depending on crystal orientation), which could be explained by presence of an impurity or other defect absorption band.

\subsection{PL emission and excitation}

Characteristic features of PL at $10 \mathrm{~K}$ are presented by emission and excitation spectra in Figs. 2 and 3, correspondingly. These spectra are measured on UV-VIS experimental set-up, using a deuterium lamp as a source of excitation light. At low temperatures $(<140 \mathrm{~K})$ the emission spectrum contains four main bands at $4.43 \mathrm{eV}(280 \mathrm{~nm}), 3.76 \mathrm{eV}(330 \mathrm{~nm})$, $2.38 \mathrm{eV}(520 \mathrm{~nm})$ and $1.77 \mathrm{eV}(700 \mathrm{~nm})$. Such set of luminescence bands coincides well with the emission bands observed in cathodoluminescence spectrum at RT [14]. Heating of the sample to temperatures up to $700 \mathrm{~K}$ and

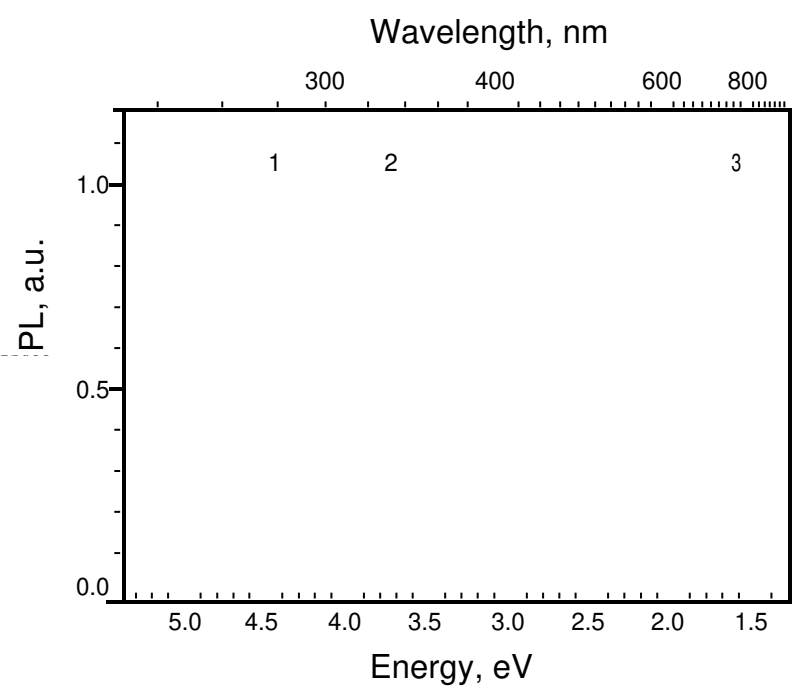

Fig. 2. Photoluminescence of $\mathrm{LiGaO}_{2}$ under deuterium lamp excitation $6.42 \mathrm{eV}$ (1), $5.85 \mathrm{eV}$ (2), $4.96 \mathrm{eV}(3)$ at $10 \mathrm{~K}$.

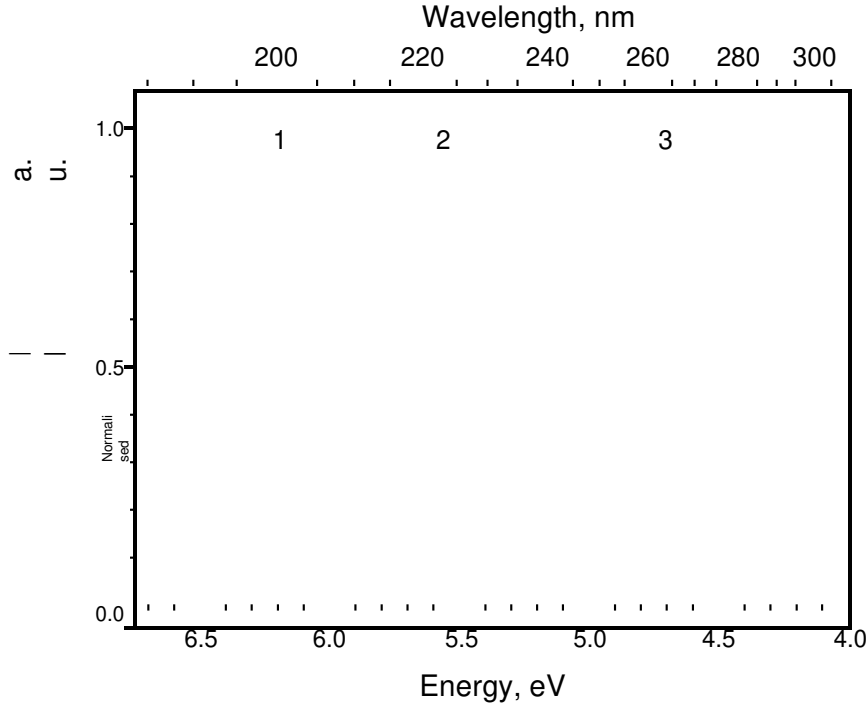

Fig. 3. Photoluminescence excitation of $\mathrm{LiGaO}_{2}$ for emission $4.43 \mathrm{eV}(1), 3.76 \mathrm{eV}$ (2), $1.77 \mathrm{eV}(3)$ at $10 \mathrm{~K}$

following cooling to RT causes redistribution of the long wave-length bands: the $2.38 \mathrm{eV}$ band increases and the $1.77 \mathrm{eV}$ band decreases, probably due to thermal restructuration of intrinsic defects. Excitation of the $4.43 \mathrm{eV}$ emission band seems to form an excitation band with maximum $6.29 \mathrm{eV}$ (see Fig. 3, curve 1), which is located inside the fundamental absorption region; the observed shape of this excitation band is not completely reliable because the spectral region below $6.2 \mathrm{eV}$ lies on the brink of sensitivity capa-bilities of the equipment, taking into account the weak excitation light. The excitation band's apparent tail at $5.9 \mathrm{e} 5.4 \mathrm{eV}$ is explained by interference with the 3.76 $\mathrm{eV}$ band due to use of wide mono-chromator slits when monitoring the weak luminescence signal. Excitation of the $3.76 \mathrm{eV}$ emission band forms a band, centred around $5.8 \mathrm{eV}$ (Fig. 3, curve 2), while both 2.38 and $1.77 \mathrm{eV}$ emission bands are excited in the $4.9 \mathrm{eV}$ excitation band (Fig. 3, curve 3). The partial overlapping of the $4.43 \mathrm{eV}$ emission band with the $4.9 \mathrm{eV}$ excitation band makes possible the reabsorption of $4.43 \mathrm{eV}$ light by the excitation band of the $1.77 \mathrm{eV}$ emission.

Increase of temperature causes thermal quenching of all emis-sion bands, but quenching rate of the $4.43 \mathrm{eV}$ band is the fastest. In the case of the deuterium lamp excitation the $4.43 \mathrm{eV}$ emission band together with its excitation band disappears at temperature $140 \mathrm{~K}$, while the rest bands survive at RT and higher temperatures. At RT under lamp excitation the luminescence spectrum contains the 3.76, 2.38 and $1.77 \mathrm{eV}$ bands. Arrhenius plot of the thermal behaviour of luminescence bands excited by deuterium lamp (not shown graphically) gives the following values for the activation energies: 47 , 43 and $52 \mathrm{meV}$ for the 4.43, 3.76 and $1.77 \mathrm{eV}$ emission bands, correspondingly. Under high excitation intensity, which will be described later, activation energy of the $4.43 \mathrm{eV}$ band is $86 \mathrm{meV}$.

Use of the VUV experimental set-up allows measurement of excitation spectra at much higher photon energies compared to UV-VIS set-up, covering the spectral region of $\mathrm{LiGaO}_{2}$ fundamental absorption. The excitation sources of the VUV set-up include ArF (193 nm, or $6.42 \mathrm{eV}$ ) and F2 (157 nm, or 7.9 $\mathrm{eV}$ ) lasers and a deuterium lamp, and the temperature range is limited by $60 \mathrm{e} 300 \mathrm{~K}$. At low temperatures PL emission spectrum has the same 4.43, 3.76, 2.38 and $1.77 \mathrm{eV}$ bands under all excitation sources, no additional emission was observed. At RT the $4.43 \mathrm{eV}$ emission band is seen only under laser excitation.

PL excitation spectra measured at $80 \mathrm{~K}$ are shown in Fig. 4, for 


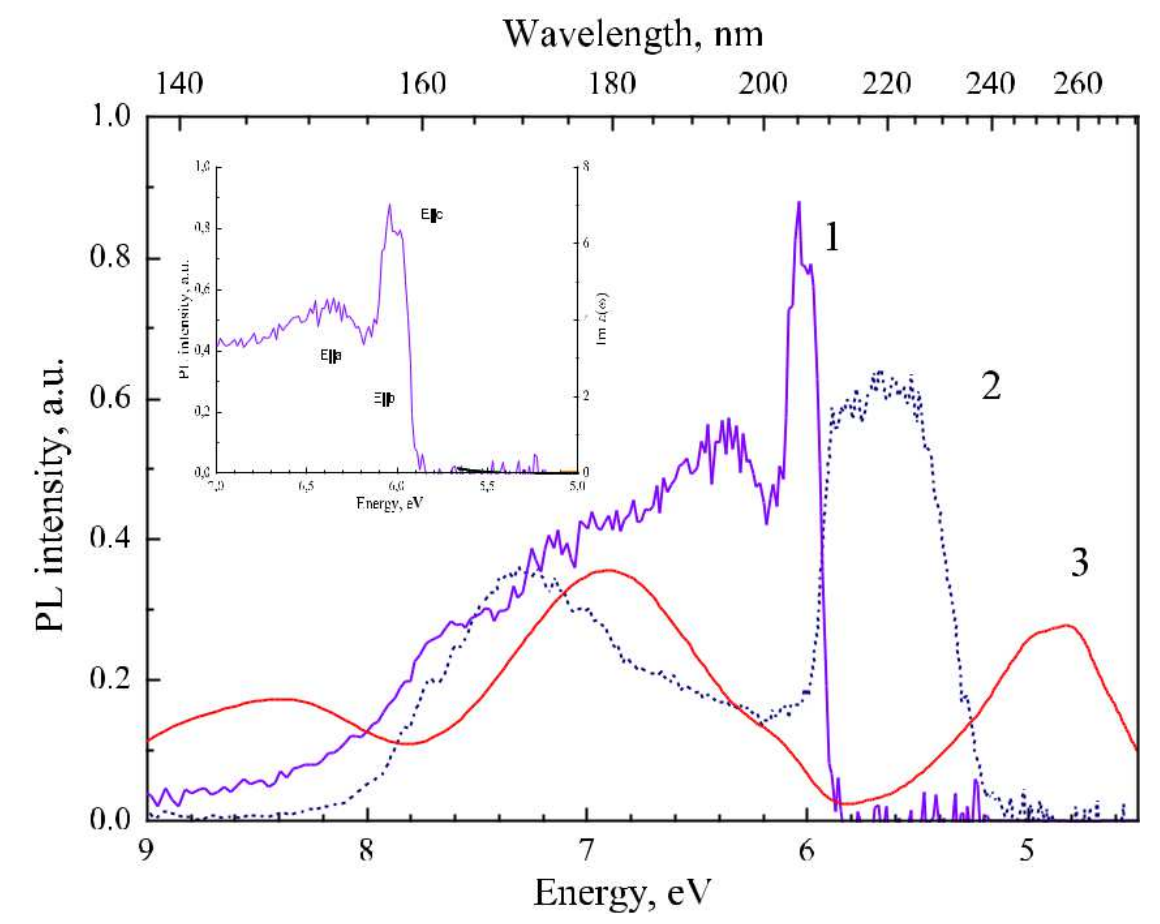

Fig. 4. Photoluminescence excitation spectra in VUV region at $80 \mathrm{~K}$ for emission bands $4.43 \mathrm{eV}$ (1), $3.76 \mathrm{eV}$ (2) and $1.77 \mathrm{eV}$ (3). Insert: comparison of the excitation spectrum of the $4.43 \mathrm{eV}$ band with imaginary part of dielectric functions for polarizations along the $\mathrm{a}, \mathrm{b}$ and $\mathrm{c}$ crystallographic axes, data taken from Ref. [17].

this measurement the emission bands were selected by optical glass filters or interference filters. Excitation spectrum of the $4.43 \mathrm{eV}$ emission band (Fig. 4, curve 1) is presented with a broad band of a complex structure in the $6 \mathrm{e} 9 \mathrm{eV}$ range with a sharp feature around $6 \mathrm{eV}$ standing apart. The excitation subband at $6 \mathrm{eV}$ practically coincides with the curves of imaginary part of dielectric functions for polarizations along the a, b and c crystallographic axes (see insert of Fig. 4) based on results of ellipsometric measurements and assigned to exciton absorption [17]. Excitation of the $4.43 \mathrm{eV}$ emission band is located entirely in the spectral range of funda-mental absorption, no additional excitation bands are observed in the band gap region. Structure of the excitation spectrum most probably corresponds to superposition of exciton and band-to-band transitions of $\mathrm{LiGaO}_{2}$ crystal.

Excitation spectrum of the $3.76 \mathrm{eV}$ (Fig. 4, curve 2) in addition to the 5.8 $\mathrm{eV}$ band contains also features in the VUV region, with the dominant subband at $7.4 \mathrm{eV}$, while the excitation spectrum of the

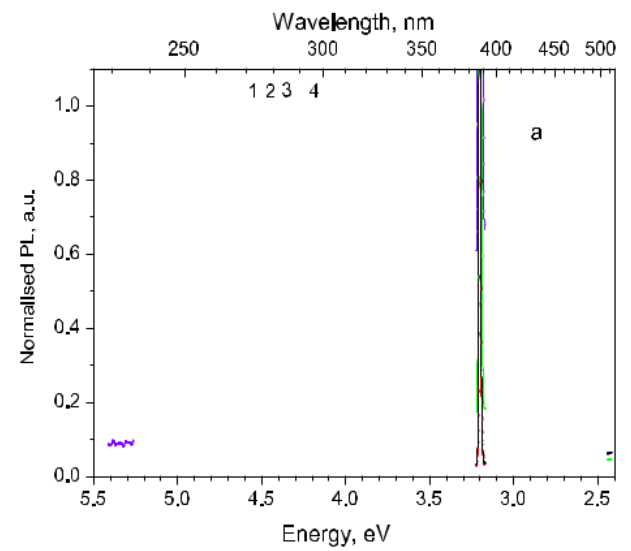

integrated $2.4 \mathrm{e} 1.8 \mathrm{eV}$ emission presented by curve 3, consists of the already mentioned $4.9 \mathrm{eV}$ band, and two subbands in VUV region e 6.05 and 7.29 $\mathrm{eV}$. Bands in below bandgap excitation region correspond to photo-ionisation processes of luminescence centres. Latter processes also possess few higher energy subbands due to electron transitions from luminescence centres (which could be impurities or intrinsic defects) to higher energy conduction subbands with further thermalization.

\subsection{Effects of excitation intensity on luminescence properties}

Presence of the $4.43 \mathrm{eV}$ emission band in PL emission spectra produced by excitation with $193 \mathrm{~nm}(6.42 \mathrm{eV})$ laser and its absence under deuterium

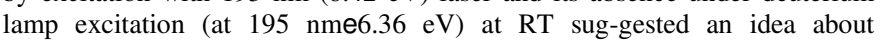
dependence of $\mathrm{LiGaO}_{2}$ PL properties on exci-tation intensity.

To examine effect of the excitation intensity on PL properties of

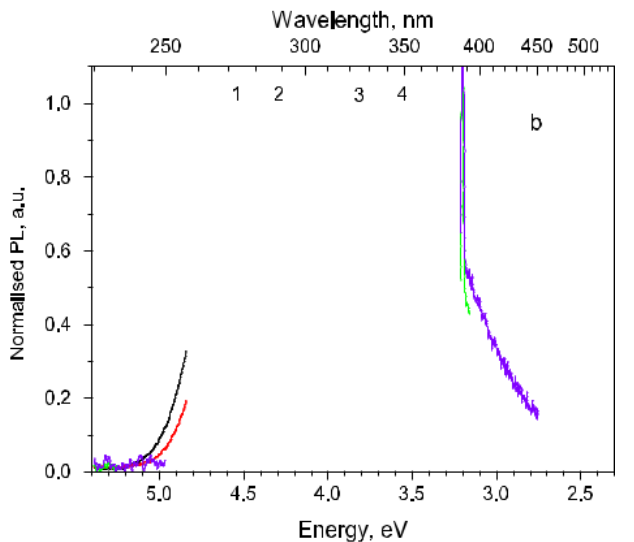

Fig. 5. PL under $193 \mathrm{~nm}\left(6.42 \mathrm{eV}\right.$ ) laser excitation with excitation intensity $50 \mathrm{~mJ} / \mathrm{cm}^{2}$ (a) and $0.3 \mathrm{~mJ} / \mathrm{cm}^{2}$ (b), at temperatures $10 \mathrm{~K}(1), 100 \mathrm{~K}(2), 200 \mathrm{~K}$ (3) and $300 \mathrm{~K}$ (4). Line at $3.20 \mathrm{eV}$ is the second order of laser excitation line. 
$\mathrm{LiGaO}_{2}$ we have measured PL spectra in identical conditions under $193 \mathrm{~nm}$ $(6.42 \mathrm{eV})$ laser excitation with varied intensity at $10 \mathrm{e} 300 \mathrm{~K}$ temperatures. In Fig. 5 there is shown the short wavelength part of the normalised emission spectra containing the 4.43 and $3.76 \mathrm{eV}$ emission bands. At $10 \mathrm{~K}$ the spectra are similar; they are repre-sented with the $4.43 \mathrm{eV}$ band notwithstanding excitation intensity (Fig. 5a, curve 1 and b, curve 1). Rise of temperature causes decrease of relative contribution of the $4.43 \mathrm{eV}$ band and increase of the $3.76 \mathrm{eV}$ band. From comparison of two PL spectra sets it is seen that thermal evolution of spectra evidently depends on excitation in-tensity: the lower excitation intensity - the faster thermal quenching of the $4.43 \mathrm{eV}$ band and building-up of the $3.76 \mathrm{eV}$ band's contribution. As a result in the RT spectra obtained under the lowest excitation intensity the $4.43 \mathrm{eV}$ band is nearly not observed (see Fig. 5b, curve 4). Similar situation was observed in PL spectra excited by very weak light from deuterium lamp.

Dependence of the $4.43 \mathrm{eV}$ band intensity on temperature for different excitation cases is shown in Fig. 6. The curves are nor-malised to maximum intensity. Two regions are observed in ther-mal evolution of all dependences: 1) growth until maximum value is reached and 2) thermal quenching. The lamp-excitation case stands apart, characterised with turning point at $70 \mathrm{~K}$ and complete disappearance at $150 \mathrm{~K}$. Almost parallel laser-excitation curves demonstrate a shift of turning point to higher temperatures ac-cording to excitation intensity increase $e$ the higher excitation in-tensity, the higher temperature of the turning point and higher temperature of the $4.43 \mathrm{eV}$ band's survival. Activation energy for the $4.43 \mathrm{eV}$ band under the highest excitation intensity constitutes $86 \mathrm{meV}$.

Besides, increase of excitation intensity causes a blue shift of the $4.43 \mathrm{eV}$ band's spectral position. Fig. 7 shows normalised PL spectra for 3 different intensities of the $193 \mathrm{~nm}(6.42 \mathrm{eV})$ laser light at $60 \mathrm{~K}$ e temperature, at which shape of the $4.43 \mathrm{eV}$ band is not distorted by presence of the $3.76 \mathrm{eV}$ band. The observed spectral shift con-stitutes around $0.1 \mathrm{eV}$.

\subsection{PL kinetics and time-resolved spectra}

PL kinetics was measured in the $80 \mathrm{e} 300 \mathrm{~K}$ temperature range. Measured luminescence decay time was limited by nanosecond range due to exciting pulse duration of $\operatorname{ArF}(193 \mathrm{~nm}$, or $6.42 \mathrm{eV})$ and $\mathrm{F} 2(157 \mathrm{~nm}$, or $7.9 \mathrm{eV})$ constituting $5 \mathrm{~ns}$.

The $4.43 \mathrm{eV}$ decay curve has a complex nonexponential shape;

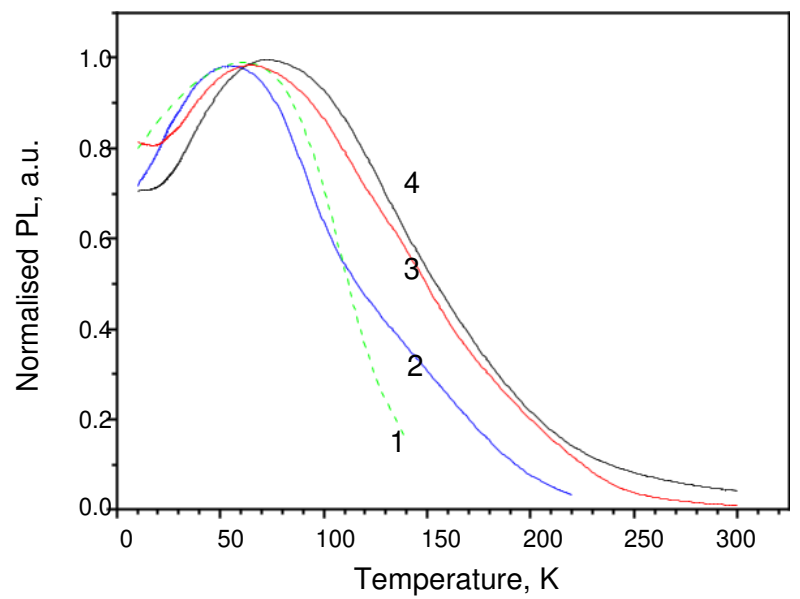

Fig. 6. Dependence of $4.43 \mathrm{eV}$ emission band intensity on temperature, for excitation using 1) $6.29 \mathrm{eV}$ light from deuterium lamp selected by monochromator; and $193 \mathrm{~nm}(6.42 \mathrm{eV})$ laser light with intensity 2) $\left.0.3 \mathrm{~mJ} / \mathrm{cm}^{2}, 3\right) 3.5 \mathrm{~mJ} / \mathrm{cm}^{2}$, 4) $50 \mathrm{~mJ} / \mathrm{cm}^{2}$.

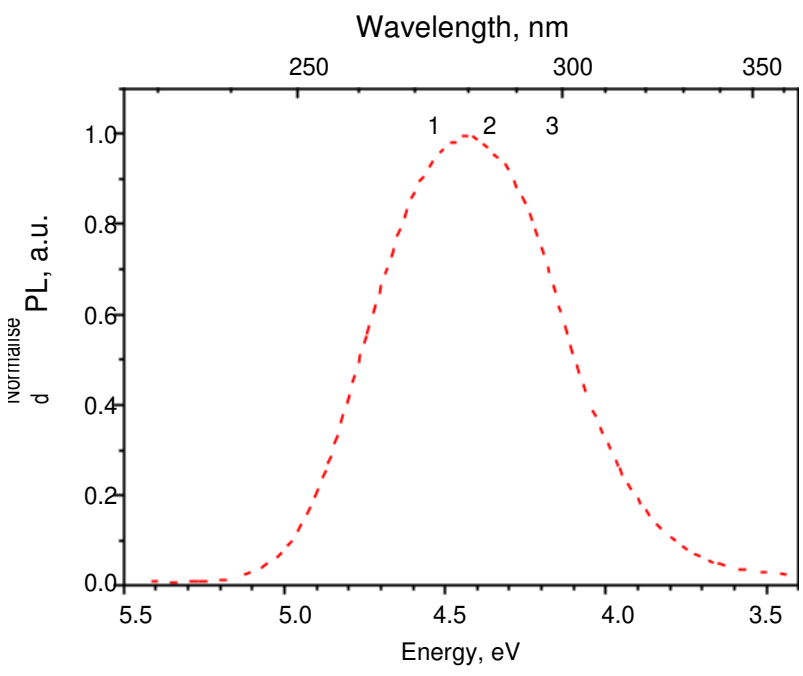

Fig. 7. $4.43 \mathrm{eV}$ emission band under $193 \mathrm{~nm}(6.42 \mathrm{eV})$ laser excitation with intensity 50 (1), 3.5 (2) and $0.3(3) \mathrm{mJ} / \mathrm{cm}^{2}$ at temperature $60 \mathrm{~K}$.

at $80 \mathrm{~K}$ it consists of 3 components with decay time of: 1) several nanoseconds; 2) several hundreds of nanoseconds and 3) several hundreds of microseconds. The second and third decay compo-nents are demonstrated in Figs. 8 and 9, correspondingly. PL decay pulses are almost independent of the laser used. Approximation of the slowest component, showed in Fig. 9 fits the law I $\sim \mathrm{t}^{1.1}$ describing dependence of luminescence intensity I on decay time $t$, which is typical for the case of tunnel luminescence [24]. With temperature rise up to RT all components become faster e the fastest component reaches 2 ns and disappears, the second component reaches several tens of nanoseconds and the slowest component e several tens of microseconds.

Decay pulse of the $3.76 \mathrm{eV}$ band is also represented with a complex nonexponential curve comprised of microsecond and millisecond components, whose duration become slightly shorter with temperature rise from 80 to $300 \mathrm{~K}$.

No effect of excitation intensity on luminescence decay time was observed.

Time resolved spectra of $\mathrm{LiGaO}_{2}$ excited by the $193 \mathrm{~nm}(6.42 \mathrm{eV})$ laser at $80 \mathrm{~K}$ is shown in Fig. 10 in comparison with the steady-state spectrum, presented by the $4.43 \mathrm{eV}$ band with a tail in the long wavelength side. PL pulses with $3 \mathrm{~ns}, 3 \mathrm{~ms}$ and $80 \mathrm{~ms}$ were chosen for

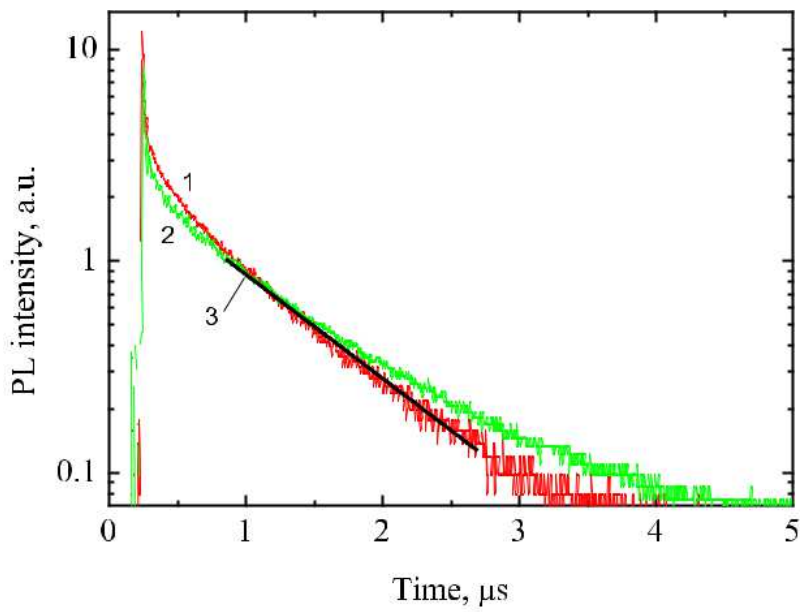

Fig. 8. PL decay of $\mathrm{LiGaO}_{2}$ (second component) at $80 \mathrm{~K}$ under excitation with $193 \mathrm{~nm}(6.42$ $\mathrm{eV}$ ) laser (1) and $157 \mathrm{~nm}(7.9 \mathrm{eV})$ laser (2), fit $\mathrm{z} 900 \mathrm{~ns}$ is shown by line (3). 


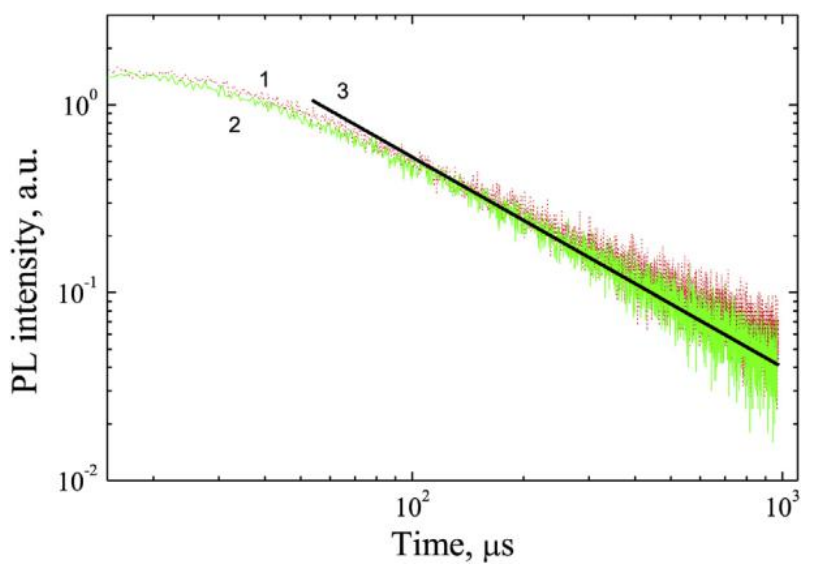

Fig. 9. PL decay of $\mathrm{LiGaO}_{2}$ (the slowest component) at $80 \mathrm{~K}$ under excitation with $193 \mathrm{~nm}$ $(6.42 \mathrm{eV})$ laser (1) and $157 \mathrm{~nm}(7.9 \mathrm{eV})$ laser (2), fit $\mathrm{I} \sim \mathrm{t}^{1.1}$ is shown by line (3).

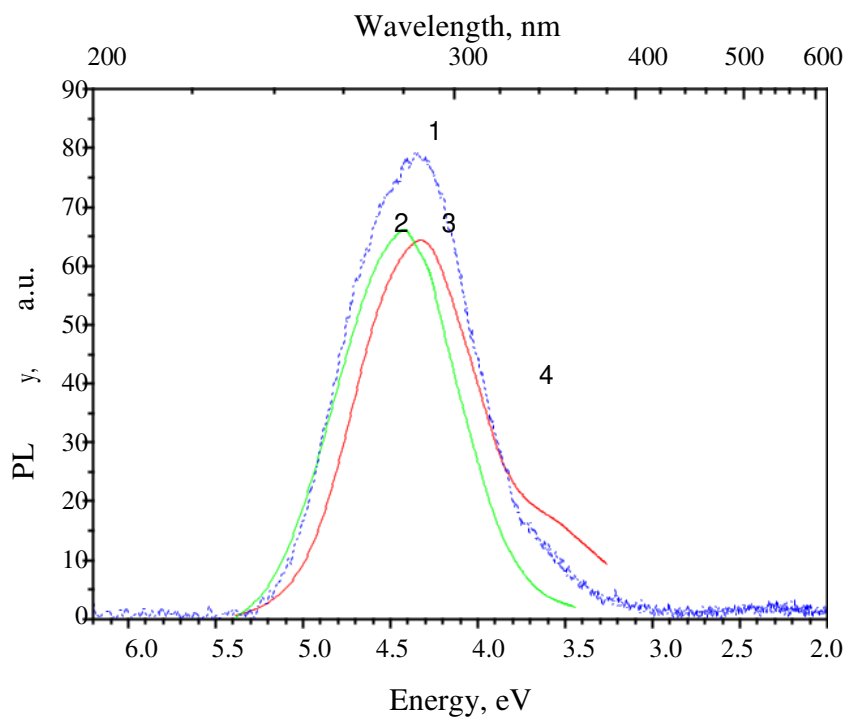

Fig. 10. Time resolved PL spectra of $\mathrm{LiGaO}_{2}$ under $193 \mathrm{~nm}(6.42 \mathrm{eV})$ laser excitation at $80 \mathrm{~K}$ detected in steady state mode (1), and selecting PL pulses with decay time $3 \mathrm{~ns}$ (2), 3 ms (3) and $80 \mathrm{~ms}$ (4).

spectral depiction. Spectral distribution of PL pulses with decay time $80 \mathrm{~ms}$ corresponds to the $3.76 \mathrm{eV}$ band (Fig. 10, curve 4 )

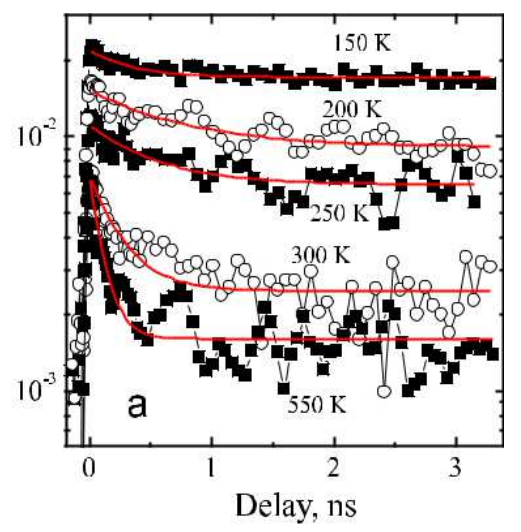

responsible for the tail of the emission spectra. Spectral distribution of the 3 ns and $3 \mathrm{~ms}$ pulses forms two subbands inside the steady-state $4.43 \mathrm{eV}$ band (Fig. 10, curve 1); the "faster" subband (Fig. 10, curve 2) is blue-shifted compared to the "slower" subband (Fig. 10, curve 3) by $0.1 \mathrm{eV}$.

$\mathrm{LiGaO}_{2}$ revealed luminescence afterglow, which was better seen after X-ray irradiation. Afterglow luminescence detected in the range of tens of seconds at $60 \mathrm{~K}$, was fitted by the laws $\mathrm{I} \sim \mathrm{t}^{0.8}$ and $\mathrm{I} \sim \mathrm{t}^{1.2}$ for the 4.43 and $3.76 \mathrm{eV}$ emissions, correspondingly.

\subsection{Pump-probe experiments}

In pump-probe experiments including measurements of differ-ential transmittivity and light induced transient grating decay excitation was done by picosecond laser with emission wavelength $213 \mathrm{~nm}(5.82 \mathrm{eV})$, which corresponds to the edge of the band gap and in the same time falls into excitation band of the $3.76 \mathrm{eV}$ emission.

Measurements of Differential transmittivity characterises change of absorption corresponding to laser excitation pulse caused excess carrier density, which could be either free or trapped.

Differential transmission decays measured at different temper-atures were fitted with two-exponential functions DT $1 / 4 \mathrm{~A} 1 \exp \left(-\mathrm{t} / \mathrm{t}_{1}\right) \mathrm{p}$ A2 $\exp \left(-\mathrm{t} / \mathrm{t}_{2}\right)$ (Fig. 11a). Fast decay component ( $\left.t_{1} 1 / 4100 \mathrm{e} 700 \mathrm{ps}\right)$ and slow one ( $\left.\mathrm{t}_{2}>4 \mathrm{~ns}\right)$ were observed. The fast component appears due to absorption by free carriers (presumably electrons) of probe photons and subsequent scattering of free carriers to en-ergy bands with help of optical phonons. Slow component is due to photoionisation of shallow donors by probe photons, which were quickly filled ( $\mathrm{z} 1 \mathrm{~ns})$ by electrons after excitation; photoionized electrons are scattered to higher energy bands with help of optical phonons. Holes shortly after excitation are quickly captured to shallow acceptors and further are being slowly released, they recombine with electrons through nonradiative midgap defects and provide slow decay (electrons cannot recombine non-radiatively if holes are not released and captured to midgap defects).

Fast component amplitude $\mathrm{A}_{1}$ increases with temperature as $\sim \mathrm{T}^{1 / 2}$, which corresponds to temperature dependence of ab-sorption cross section on optical phonons [25]. Fast component lifetime decreased with temperature approximately as $\mathrm{T}^{1}$ (Fig. 11b), indicating possible increase of carrier capture cross sec-tion with temperature and/or density of electrically active traps due to their ionisation [25]. Thermal activation energy of $E_{a} 1 / 4104 \mathrm{meV}$ was found from slow trapped carrier component $\mathrm{A}_{2}$ differential transmission signal decrease with temperature. $\mathrm{A}_{2}$ was fitted with

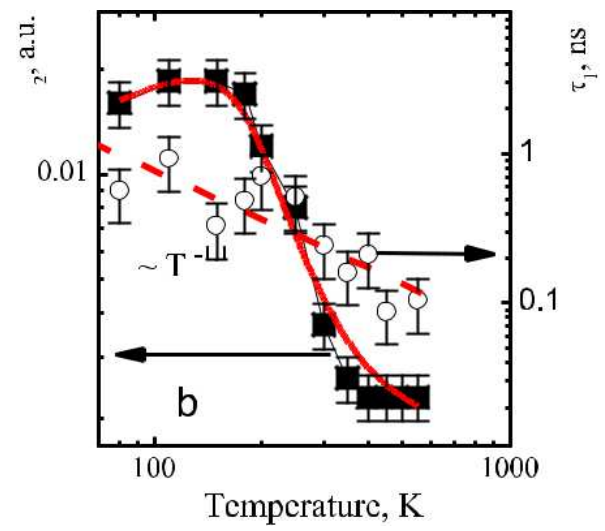

Fig. 11. Characteristics of $\mathrm{LiGaO}_{2}$ differential transmission: (a) differential transmission decays at different temperatures and (b) temperature dependences of slow component amplitude and fast component decay time, which slope is fitted as $t_{1} \sim \mathrm{T}^{1.1}$ 
const* $T^{1 / 2} /\left(1 \quad p \quad a^{*} \exp \left(-E_{a} / k T\right)\right)$ relationship, where $T^{1 / 2}$ corresponds to electron absorption cross section on optical phonons tempera-ture dependence. Latter explains DT signal increase in $80 \mathrm{e} 100 \mathrm{~K}$ range. Activation energy pointed out to activation of presumably shallow acceptors (probably responsible for $4.43 \mathrm{eV}$ band, having similar activation energy and temperature dependence, see Fig. 6). Remaining slow component part at the highest temperatures (DT 1/4 0.0015) should indicate for deeper defects (e.g. related to $3.76 \mathrm{eV}$ band).

Simultaneously with differential transmittivity the pump laser induced luminescence was recorded. It was found that the carriers generated in pumpprobe experiments were quickly captured to impurities or native defects leading to dominant $3.76 \mathrm{eV}$ defect emission as $213 \mathrm{~nm}(5.82 \mathrm{eV})$ excitation corresponds to excitation of this emission band. Carrier density of $10^{1819} \mathrm{~cm}$ 3 was esti-mated from $3.76 \mathrm{eV}$ band saturation: $\mathrm{N}_{\text {trap }} 1 / 4 \mathrm{aI}$ sat $/ \mathrm{hn}$. Latter trap DT decay was slow.

Light induced transient grating decay characterises free elec-trons excitation and their recombination through diffraction effi-ciency (DE). The LITG measurements showed considerably fast diffraction efficiency decays: their lifetime reduced from 200 to 30

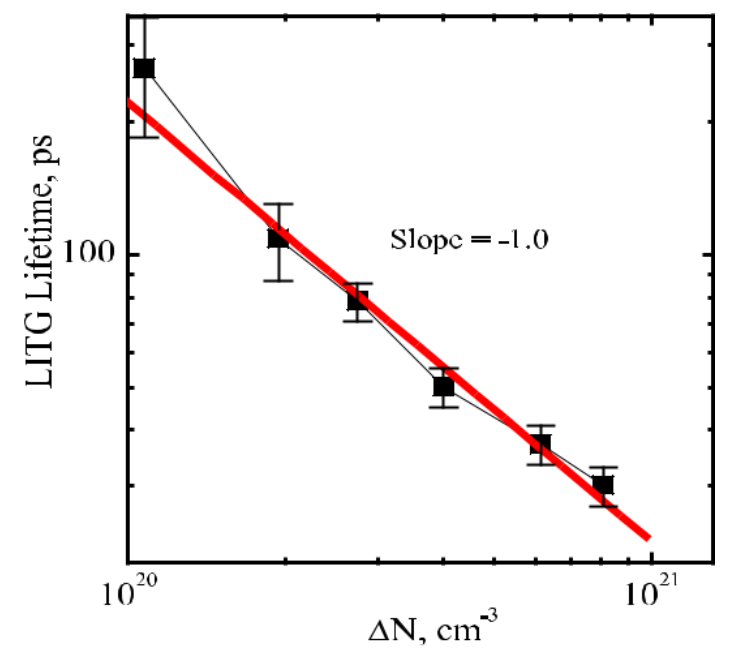

Fig. 12. Recombination lifetime dependence on carrier density DN determined by LITG. ps with excitation increase (Fig. 12). DE decay fast component lifetime at low excitation intensity coincides with that from DT fast component measurements. At low excitation intensities the fast free carrier relaxation could be explained by nonradiative capture of carriers to traps connected with defects or impurities, followed by $4.43 \mathrm{e} 3.76 \mathrm{eV}$ luminescence with much slower decays (see Fig. 11a). At higher excitations linear decrease of carrier lifetime with excited carrier density DN can be explained by trap assisted Auger recombination [26] through midgap defects. $\mathrm{In}_{\mathrm{LiGaO}}$ midgap defects are responsible for the $2.38 \mathrm{e} 1.77 \mathrm{eV}$ emission bands. The trap assisted Auger recombination process presumably involves two free electrons and a hole bound to a close midgap defect.

\subsection{Thermoluminescence}

It was found that $\mathrm{LiGaO}_{2}$ reveals thermoluminescence after preliminary irradiation with UV light. TL is excited in the region of PL excitation bands $(4.5 \mathrm{e} 6 \mathrm{eV})$ and the most efficiently in the band-to-band transitions region $(6 \mathrm{e} 8.5 \mathrm{eV})$. For TL measurements in the $10 \mathrm{e} 300 \mathrm{~K}$ range irradiation was done with light corresponding to the PL excitation bands: at $6.26,5.59 \mathrm{eV}$ (shown in Fig. 13a) and $4.96 \mathrm{eV}$. At all irradiation wavelengths TL curves have 2 main TL peaks at 120 and $170 \mathrm{~K}$ (Fig. 13a). In TL emission the 2.38 and $1.77 \mathrm{eV}$ bands are dominant, the $3.76 \mathrm{eV}$ band is hardly seen, and the $4.43 \mathrm{eV}$ band is not observed e see Fig. 13b. Spectral distribution of TL response varies with temperature and depends on particular irradiation wavelength. The tentative study of $\mathrm{TL}$ at elevated tem-peratures after preliminary irradiation at RT implies the presence of an intensive peak around $400 \mathrm{~K}$ with TL emission in $2.4 \mathrm{e} 1.5 \mathrm{eV}$ spectral region.

\subsection{PL polarization}

PL polarization study of $\mathrm{LiGaO}_{2}$ showed that PL reveals polari-zation almost in all emission bands in the $10 \mathrm{e} 300 \mathrm{~K}$ temperature range. Fig. 14 shows PL spectra measured at parallel (solid line) and perpendicular (dotted line) orientations of the analyser at $10 \mathrm{~K}$ (Fig. 14a) and $300 \mathrm{~K}$ (Fig. 14b). Spectral dependences of polarization degree $\mathrm{P}$ calculated according to formula (1) at 10 and $300 \mathrm{~K}$ are shown on (Fig. 14c and d, correspondingly). Luminescence polari-zation was not observed in the $4.43 \mathrm{eV}$ band, which occurs under
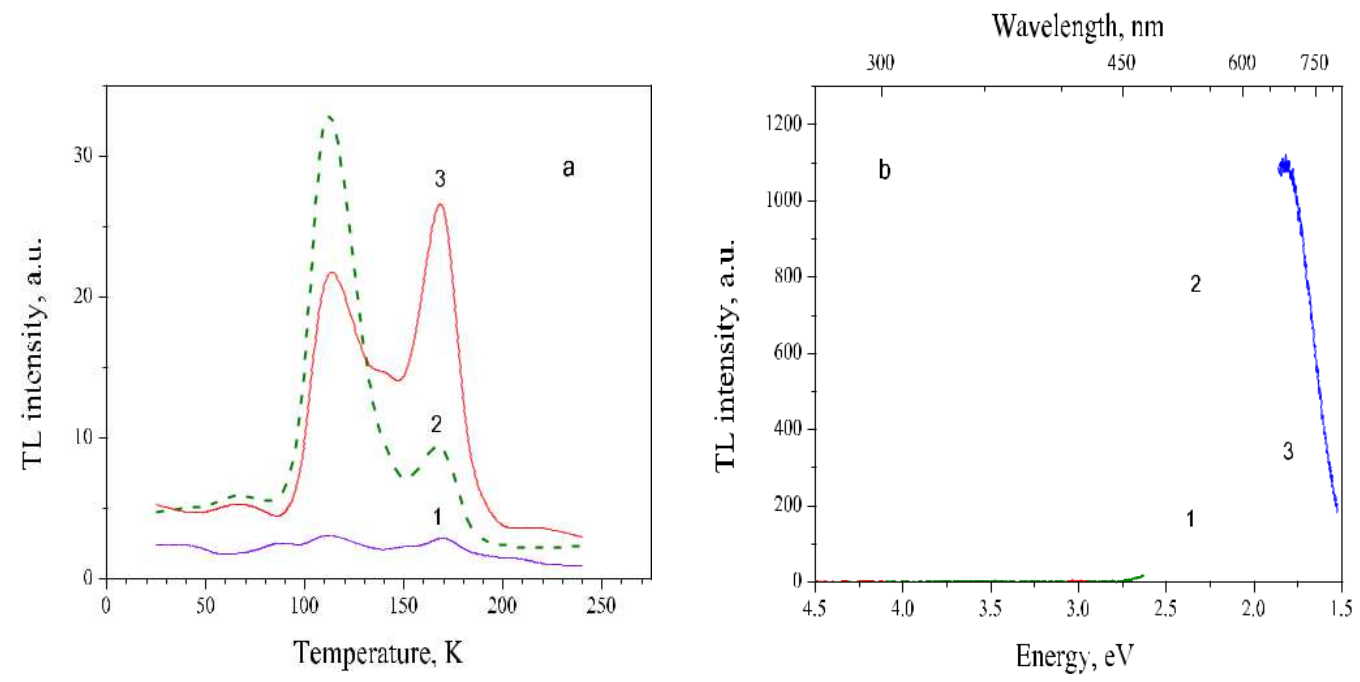

Fig. 13. a) TL curves after irradiation with $5.59 \mathrm{eV}$ light at $10 \mathrm{~K}$ for emission selecting $3.76 \mathrm{eV}$ (1), $2.38 \mathrm{eV}(2), 1.77 \mathrm{eV}(3)$ and b) TL emission spectra at temperatures $105 \mathrm{~K}(1), 120 \mathrm{~K}(2), 170 \mathrm{~K}$ (3). 

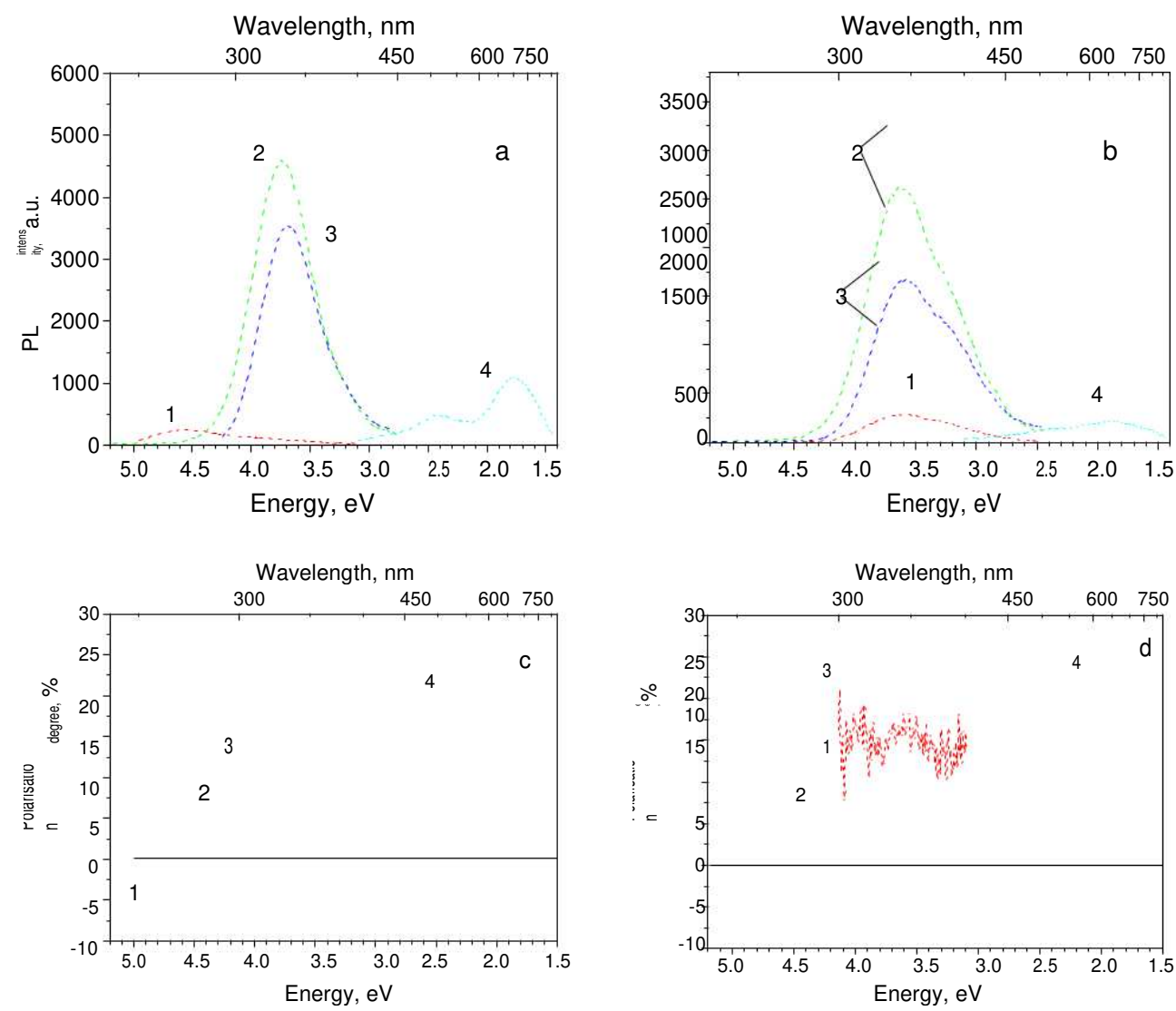

Fig. 14. Polarised PL measured at parallel (solid line) and perpendicular (dotted line) orientations of analyser at $10 \mathrm{~K}$ (a) and $300 \mathrm{~K}$ (b) and spectral dependences of polarization degree at $10 \mathrm{~K}$ (c) and $300 \mathrm{~K}$ (d) under excitation $6.2 \mathrm{eV}(1), 5.74 \mathrm{eV}(2), 5.46 \mathrm{eV}$ (3) and $4.96 \mathrm{eV}$ (4).

deuterium lamp excitation $6.2 \mathrm{eV}$ only at low temperature (Fig. 14a, curve 1), while all other bands demonstrated rather high polari-zation degree up to $20 \mathrm{e} 25 \%$. Character of spectral dependence of $\mathrm{P}$ within the emission bands $4.2 \mathrm{e} 2.9 \mathrm{eV}$ and $2.7 \mathrm{e} 1.5 \mathrm{eV}$ speaks in favour of complex nature of the corresponding emission, contain-ing several subbands.

\section{Discussion}

Different experimental methods have been used to explore processes caused by light in lithium metagallate $\mathrm{LiGaO}_{2}$. The pump-probe methods brought information about excitation and relaxa-tion of charge carriers, while photoluminecence, thermolumines-cence and polarised luminescence identified luminescence processes in this material. Luminescence spectra of $\mathrm{LiGaO}_{2}$ contain 4 main emission bands peaking at 4.43, 3.76, 2.38 and 1.77 $\mathrm{eV}$. Their presence in TL emission spectra and/or luminescence afterglow testifies that all these emissions are due to recombination pro-cesses. Let us discuss the observed luminescence bands in more detail.

Discussing the luminescence mechanisms we will use a concept of donors and acceptors. Free charge carriers e electrons and holes, which are generated during excitation are seized on electron and hole trap levels, creating donor and acceptor centres, correspond-ingly. Donor and acceptor centres participate in recombination processes. Depending on generation process the recombining donor-acceptor pairs are characterised by either random distribution of separation distance between components (random DAP) or close separation distance between genetically related pair com-ponents (geminate DAP).
The $4.43 \mathrm{eV}$ band is excited only at energies at and above $6 \mathrm{eV}$ e in the spectral range corresponding to exciton states and band-to-band transitions. The excitation spectrum of this band (Fig. 4) containing a sharp feature at 6 $\mathrm{eV}$, which is assigned to exciton absorption [17] allows assuming exciton character of the emission band. However, the further speculations based on experimental results show the $4.43 \mathrm{eV}$ band is due rather to donor acceptor radiative recombination than exciton (free or bound) emission.

Properties of the $4.43 \mathrm{eV}$ emission band depend on excitation: rise of excitation intensity causes blue shift of the band's maximum (Fig. 7) and decrease of the thermal quenching rate e the emission band survives at higher temperature (Figs. 5 and 6). Kinetic mea-surements of PL demonstrate a complex shape of $4.43 \mathrm{eV}$ decay consisting of superposition of exponents with time constants from nanosecond to microsecond range (Figs. 8 and 9) and X-ray caused afterglow decay time reaches several tens of seconds. Fitting of the decay of PL signal and afterglow gives the law close to $\mathrm{I} \sim \mathrm{t}^{1}$, which is typical for tunnel luminescence. Time resolved spectra show that the luminescence signal of $4.43 \mathrm{eV}$ emission with the faster decay time is blue shifted by $\sim 100 \mathrm{meV}$ compared to that with the slower decay time (Fig. 10).

All these facts allow conclusion that the $4.43 \mathrm{eV}$ band is caused by tunnel luminescence of donor-acceptor pairs with random dis-tribution of separation distance between components. Excitation with UV with photon energies $>6$ $\mathrm{eV}$ in $\mathrm{LiGaO}_{2}$ generates (through excitonic processes or/and band-to-band transitions) a set of do-nors and acceptors with different separation distances. Rise of excitation intensity causes increase of concentration of donors and acceptors and decrease of the mean separation distance $r$ between DAP components. It leads to increase of the recombination 
emission energy, described by expression [27]:

$$
\text { EðrP } 1 / 4 E_{g a p} E_{a} p E_{d} P p e^{2} \varepsilon r ;
$$

where $E_{g a p}$ is the band gap energy, $E_{a}$ and $E_{d}$ are the $A$ and $D$ binding energies, $\varepsilon$ is dielectric constant and $r$ is the D-A separation dis-tance. The estimation shows, that the $\sim 100 \mathrm{meV}$ blueshift would provide donor density of $\mathrm{ND} \sim \mathrm{r}^{3} \sim 10^{19} \mathrm{~cm}^{3}$. The closer DAPs better resist thermal quenching providing luminescence survival at higher temperatures. Separation distance between donors and ac-ceptors determines also recombination luminescence decay time; tunnel recombination between the closer pairs corresponds to faster kinetics and higher recombination emission energy, explaining the results of time-resolved spectra.

There may be also another reason partially contributing to blueshift: at the highest excitation intensities free electron to acceptor (eA) recombination process can occur with a fast decay $(\sim 1 \mathrm{~ns})$. If so, the difference of the $4.43 \mathrm{eV}$ emission band's activa-tion energy at high excitation intensity $(\sim 86 \mathrm{meV})$ and low excita-tion intensity ( 47 meV) (Fig. 6) could be explained by combination of two different recombination processes. At the highest excitations the eA recombination process prevails and $86 \mathrm{meV}$ activation energy is related to acceptor. With decrease of the excitation intensity the DAP recombination becomes dominant and is described by ionisation of shallow donor (characterised with activation energy of $47 \mathrm{meV}$ and saturated at the highest excitations). Simultaneous coexistence of both recombination processes could be a reason of 3 time components of the $4.43 \mathrm{eV} \mathrm{PL}$ band decay described in 3.3 e the fast decay component can be attributed to electron-acceptor process while the slower components 2 and 3 - to the DAP processes.

Absence of polarization of the $4.43 \mathrm{eV}$ emission (see Fig. 14) confirms the idea of its origin from tunnel recombination of random DAPs, because random distribution of recombination partners without any preferential direction in respect with the crystallographic axis should not produce any polarised lumines-cence. The eA process, if present at the highest excitation in-tensities, also would provide nonpolarised luminescence. Absence of the $4.43 \mathrm{eV}$ in the TL emission spectra produced by UV light irradiation, in its turn, does not contradict recombination nature of this emission band $e$ evidently concentration of free charge carriers generated as a result of thermal release of trap centres during TL process is not sufficiently high to produce close DAPs capable of radiative recombination, similarly to case of the $4.43 \mathrm{eV}$ band's absence under low intensity lamp excitation at RT.

The microscopic origin of DAP components responsible for the $4.43 \mathrm{eV}$ emission band is not detectable from the results obtained. Acceptor component of the DAP responsible for the $4.43 \mathrm{eV}$ could be connected with oxygen vacancies, which are typical intrinsic defects in oxide semiconductors, for example, in $\mathrm{ZnO}$ [28].

The $3.76 \mathrm{eV}$ emission band is excited both in the region of fundamental (2) absorption peaking at $7.29 \mathrm{eV}$ and in its own excita-tion band at $5.8 \mathrm{eV}$, adjoining the band edge (see Fig. 4). The $5.8 \mathrm{eV}$ band is observed also in absorption spectrum as a red shift of the fundamental absorption edge, and might be connected with some intrinsic defect or impurity. Kinetic measurements show that decay of the $3.76 \mathrm{eV}$ band is complex, consisting of several components, and for all components time constants are longer than those for the $4.43 \mathrm{eV}$ band. Fitting law of the luminescence afterglow is $\mathrm{I} \sim \mathrm{t}$ 1.2

1.2 , which is close to decay law of tunnel luminescence. No dependence on excitation intensity was observed for this band. Irradiation of the sample by light from the $5.8 \mathrm{eV}$ excitation band produces TL signal, testifying release ofbands e peaking at $8.3,7.0$ and $4.9 \mathrm{eV}$ and will be dis-cussed together. These the charge carriers into conduction (or valence) bands. The $3.76 \mathrm{eV}$ emissionbands also have recombination character and are connected with some defects. The band with relatively low intensity is observed in the emission spectra of TL caused by irradiation by UV light with photon energies higher than $5.4 \mathrm{eV}$. Presence of the emission polarization with rather high polarization degree (up to
$20 \%$ ) speaks in favour of regular distribution of the recombination partners in crystallographic lattice of $\mathrm{LiGaO}_{2}$. All these experi-mental facts allow conclusion that the $3.76 \mathrm{eV}$ emission band is caused by tunnel recombination of genetically related or geminated DAPs. Origin of the luminescence centres of the $3.76 \mathrm{eV}$ emission is not clear at the moment. Slower decay of this band in comparison to that of $4.43 \mathrm{eV}$ indicates that the band could be related to deeper acceptor and the observed activation energy of the $3.76 \mathrm{eV}$ band, $\sim 43 \mathrm{meV}$, could be related to activation of donor of the geminate DAP pair.

The 2.38 and $1.77 \mathrm{eV}$ emission bands are excited in the same excitation 2.38 and $1.77 \mathrm{eV}$ bands emerge efficiently in the TL emission spectrum after irradiation in $8.3 \mathrm{e} 4.4 \mathrm{eV}$ region. Mutual relation of the 2.38 and $1.77 \mathrm{eV}$ bands varies depending on irradiation wavelength and TL peaks (Fig. 14). Impermanence of $\mathrm{TL}$ emission spectra might be caused by presence of 
localized direct transitions between shallow traps emptied during TL, and luminescence centres e components of geminate DAPs, without entering of the heat - released charge carriers into conduction/valence bands. Localized transitions could coexist with delocalised transitions implying electronic band involvement. Similar situation was observed in thermoluminescence of AlN [29]. Besides, these bands are characterised with high polarization de-gree (up to $25 \%$ ) testifying regular orientation of the luminescence centres in $\mathrm{LiGaO}_{2}$ lattice. All these experimental facts allow pro-posal that the 2.38 and $1.77 \mathrm{eV}$ bands are caused by recombination luminescence of geminate DAPs.

Basing on experimental data we have presumably assigned the $4.43 \mathrm{eV}$ band to tunnel luminescence of the random DAP, and 3.76,

2.38 and $1.77 \mathrm{eV}$ bands e to recombination of the geminate DAP. The experimental data obtained are not sufficient to make con-clusions about microscopic origin of the donor and acceptor centres participating in luminescence processes. Position of the corresponding excitation region inside or close to the fundamental ab-sorption implies that the $4.43 \mathrm{eV}$ and $3.76 \mathrm{eV}$ emission bands most probably are related to intrinsic defects of $\mathrm{LiGaO}_{2}$, while the 2.38 and 1.77 $\mathrm{eV}$ emission bands could be connected also with presence of uncontrolled impurities. In that case DAP recombination process could be followed by intercenter luminescence. At present we cannot assume any particular impurity ion as responsible for the long wavelength emission of $\mathrm{LiGaO}_{2}$ basing on literature data, because none of the few investigated impurity luminescent centres $\left(\mathrm{Cr}^{3 p}[18,19], \mathrm{Cu}^{\mathrm{p}}\right.$ [11], $\mathrm{V}^{3 \mathrm{p}}$ [4]) are characterised with emission and excitation spectra observed.

Pump-probe experiments give information on excited non-equilibrium state of charge carriers and in the same time help to judge about radiative recombination processes causing lumines-cence. Analysis of correlation between parameters of DT and LITG and those of PL allows revealing relationship between centres contributing to various emission bands.

Carrier excitation and recombination processes are essentially affected by intensity of excitation light, as shown in Fig. 15. Dependence of slow DT signal on excitation intensity coincides rather well with that of PL at $3.76 \mathrm{eV}$ indicating donor defect saturation (saturation of $3.76 \mathrm{eV}$ band is evidenced by change of $\sim 1.0$ slope to 0.4 slope). It is worth reminding that the pump laser wavelength $213 \mathrm{~nm}$ $(5.82 \mathrm{eV})$ corresponds to excitation band of the 3.76 $\mathrm{eV}$ emission band. DT signal activation energy is related to $\sim 100 \mathrm{meV}$ deep acceptor impurity, the value of activation energy being very close to that of the $4.43 \mathrm{eV}$ band. LITG signal, which is 


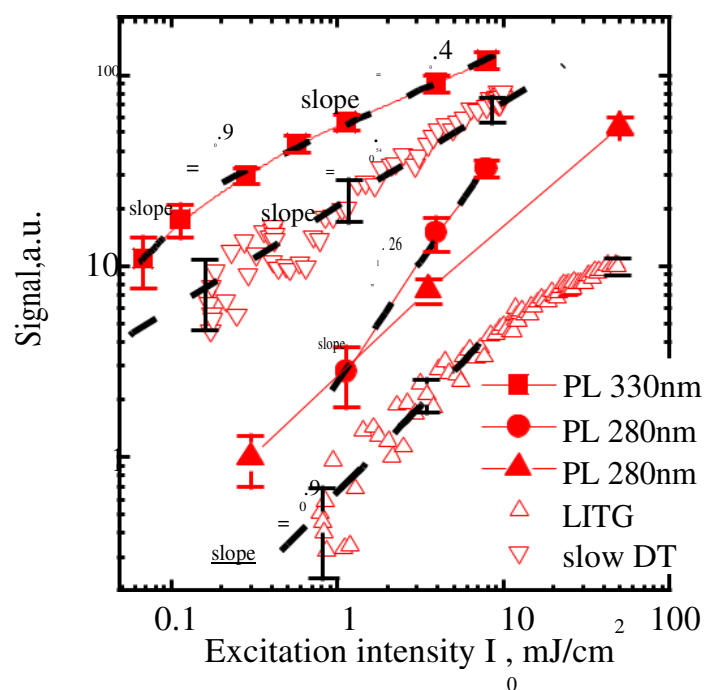

Fig. 15. Dependence of PL, DT and LITG signals on excitation intensity at $300 \mathrm{~K}$. The $3.76 \mathrm{eV}$ band saturation point is at $\sim 0.1 \mathrm{~mJ} / \mathrm{cm}^{2}$. For PL excitation $213 \mathrm{~nm}(5.82 \mathrm{eV}$ ) (squares and circles) and $193 \mathrm{~nm}(6.42 \mathrm{eV})$ (triangles) laser light was used.

equal to square root of diffraction efficiency is rather linear at low excitation intensity due to linear carrier generation, but saturates at higher excitation intensities due to reduction of carrier lifetime down to probing pulse duration of $\sim 25$ ps. Meanwhile the $4.43 \mathrm{eV}$ band PL intensity increases superlinearly (slope is $>1$ ) with $213 \mathrm{~nm}$ excitation indicating $4.43 \mathrm{eV}$ traps are being filled only when $3.76 \mathrm{eV}$ traps are saturated at $\sim 0.1 \mathrm{~mJ} / \mathrm{cm}^{2}$. Intensity increase of the $193 \mathrm{~nm}(6.42 \mathrm{eV})$ laser excitation causes linear rise of the $4.43 \mathrm{eV}$ PL band and saturation of the $3.76 \mathrm{eV}$ PL band at lower excitations because absorption coefficient is larger at $193 \mathrm{~nm}$ (see Fig. 1). Linear dependence of the $4.43 \mathrm{eV}$ PL band intensity on excitation could indicate free e to bound process with PL DN*NA. Also PL DN*const could be an indication of fast, in comparison to $193 \mathrm{~nm}$ excitation pulse duration (5 ns), recombination of close DAP pairs - in that quasistationary regime the recombined close pair density is pro-portional to excitation intensity. DT saturation is also evident, indicating that the $4.43 \mathrm{eV}$ band defects are saturated during photoneutralization because initially charged donor and acceptor impurity densities are similar due to crystal electrical neutrality.

Similar saturation of the $3.76 \mathrm{eV}$ band and the slow DT signal, and simultaneously coincident activation energies of DT slow decay and $4.43 \mathrm{eV}$ band indicates, that the shallow donors should be the same for these two emission bands. This is evidenced by very close donor activation energies: $\sim 43 \mathrm{meV}$ as derived from the $3.76 \mathrm{eV}$ band thermal activation and $\sim 47 \mathrm{meV}$ from the $4.43 \mathrm{eV}$ band thermal activation at low excitations. The fast DT component at low temperatures with $\sim 1 \mathrm{~ns}$ decay time rather well coincides with the fast TRPL decays of the $4.43 \mathrm{eV}$ band (free-to-bound regime), while the slow DT decay is attributable to the slow $4.43 \mathrm{eV} / 3.76 \mathrm{eV}$ PL bands decays (DAP regime) - see Fig. 9.

Unclear remains the reason of the unusual behaviour of the thermal dependence of the $4.43 \mathrm{eV}$ PL band (Fig. 6) and differential transmission (Fig. 11b), both of which have similar low temperature region of signal increase with temperature rise, and most probably are interrelated. Different possible factors could be mentioned as responsible for this phenomenon, though none of them is proved at present.

It can be proposed that at the lowest temperatures excitons are generated and quickly recombine without radiative emission through exciton-exciton Auger recombination $[23,30]$ - a process when collision of two excitons occurs. Also defect-assisted exciton
Auger recombination [31] could take place. Moreover, electron-hole droplets subject to fast Auger recombination also can be formed at low temperatures, similarly to silicon and diamond [32]. Under these conditions density of the trapped carriers, and hence, DAP pairs, reduces due to lower fraction of carriers available for capture to DAPs. That causes drop of intensity of the $4.43 \mathrm{eV}$ PL emission band. At higher temperatures, when excitons are not formed, PL (4.43 eV) and DT decline with temperature rise monotonously. Another reason of the unusual thermal dependence of the $4.43 \mathrm{eV}$ PL band can be connected with screening of closer DAP pairs by electrons weakly bound to shallow donors, which leads to PL signal drop at low temperatures as screening radius decreases with temperature as $\sim \mathrm{T}^{1 / 2}$ [25]. The $3.76 \mathrm{eV}$ band is supposed to be less sensitive to that effect due to higher concentration of very close geminate DAP pairs, which are less sensitive to screening. The DT drop at lower temperatures more plausibly could be due to reduction of excited carrier interaction with optical phonons.

In this work we have not observed direct manifestations of exciton luminescence, which should emerge near band edge around $6 \mathrm{eV}$. Maybe such luminescence, if any, is reabsorbed by excitation band $5.8 \mathrm{eV}$ or deep acceptor traps are in excess sup-pressing free exciton formation. If excitons are still generated at highest excitations and lowest temperatures they could participate in trap assisted Auger recombination through midgap defects making exciton emission negligibly low.

\section{Conclusions}

The comprehensive spectral study of lithium metagallate $\mathrm{LiGaO}_{2}$ crystal was done including methods of pump-probe techniques, optical absorption, photoluminescence, luminescence kinetics, thermoluminescence and polarised luminescence.

Excitation with UV light causes the main emission bands at 4.43, 3.76, 2.38 and $1.77 \mathrm{eV}$. Experimental results allow conclusion that the $4.43 \mathrm{eV}$ band is due to tunnel recombination of donor-acceptor pairs with random distribution of separation distance; at the highest excitation intensity the free electron-acceptor recombina-tion process is also possible. The 3.76, 2.38 and $1.77 \mathrm{eV}$ bands presumably arise as the result of recombination of geminate donor-acceptor pairs.

From pump-probe experiments it was found that free carrier recombination is fast $(<1 \mathrm{~ns})$ due to electron capture to abundant traps. Saturation of the slow DT component and the $3.76 \mathrm{eV}$ PL band is tentatively assigned to saturation of shallow ( $43 \mathrm{meV})$ donor traps. Similar activation energies of slow DT component $(104 \mathrm{meV})$ and the $4.43 \mathrm{eV}$ PL band (86 $\mathrm{meV}$ ) could be explained by DT decay relation to the $4.43 \mathrm{eV}$ band's acceptor. Linear electron lifetime reduction with excitation was explained by trap assisted Auger recombination.

To determine the particular microscopic defects responsible for the recombination luminescence of $\mathrm{LiGaO}_{2}$ the further studies are necessary.

\section{Acknowledgements}

The study was supported by the Latvia-Lithuania-Taiwan research project "Nonpolar ZnO thin films: growth-related struc-tural and optical properties" (Latvia: LV-LT-TW/2016/5, Lithuania: TAP LLT 02/2014, Taiwan: MOST 103-2923-M-110-001-MY3)

\section{References}

[1] J.P. Remeika, A.A. Ballman, Flux growth, Czochralski growth, and hydrother-mal synthesis of lithium metagallate single crystals, Appl. Phys. Lett. 5 (1964) 
$180 \mathrm{e} 181$.

[2] S. Nanamatsu, K. Doi, M. Takanashi, Piezoelectric, elastic and dielectric prop-erties of $\mathrm{LiGaO}_{2}$, Japn. J. Appl. Phys. 11 (1972) 816e822.

[3] S. Kuck, S. Hartung, Comparative study of the spectroscopic properties of $\mathrm{Cr}^{4 p}$ doped LiAlO2 and LiGaO2, Chem. Phys. 240 (1999) 387 e401.

[4] B.H.T. Chai, X.X. Zhang, in: Proceedings of the Conference on Advanced Solid State Lasers, OSA Technical Dig, 1995, pp. 289e291.

[5] T. Ishii, Y. Tazoh, S. Miyazawa, Single-crystal growth of $\mathrm{LiGaO}_{2}$ for a substrate of $\mathrm{GaN}$ thin films, J. Cryst. Growth 186 (1998) 409e419.

[6] J. Wang, L. Pu, G. Tang, J. Zhang, Electronic and structural characterization of $\mathrm{InN}$ heterostructures grown on b-LiGaO2(001) substrates, Vacuum 119 (2015) 106e111.

[7] T. Huang, S. Zhou, H. Teng, H. Lin, J. Wang, P. Han, R. Zhang, Growth and characterization of $\mathrm{ZnO}$ films on (001), (100) and (010) $\mathrm{LiGaO}_{2}$ substrates, J. Cryst. Growth 310 (2008) 3144e3148.

[8] J.-Y. Yu, T.-H. Huang, L. Chang, M.M.C. Chou, D. Gan, Growth behaviour of M-plane $\mathrm{ZnO}$ epilayer on (100) $\mathrm{LiGaO}_{2}$ by chemical vapour deposition, ECS Trans. 35 (6) (2011) $133 \mathrm{e} 139$.

[9] C. Chen, T. Yan, S.-H. Yu, C.-Y. Lee, C.-W. Chang, M.M.C. Chou, Microstructural and optical properties of high-quality $\mathrm{ZnO}$ epitaxially grown on a $\mathrm{LiGaO}_{2}$ substrate, $\mathrm{RSC}$ Adv. 5 (2015) 35405e35411.

[10] T. Omata, K. Tanaka, A. Tazuke, K. Nose, S. Otsuka-Yao-Matsuo, Novel wide band gap alloyed semiconductors, $\mathrm{x}\left(\mathrm{LiGaO}_{2}\right) 1 / 2-(1-\mathrm{x}) \mathrm{ZnO}$, and fabrication of their thin films, Sci. China Ser. E-Tech. Sci. 52 (1) (2009) 111e115.

[11] M.S. Holston, I.P. Ferguson, N.C. Giles, J.W. McClory, D.J. Winarski, J. Ji, Green luminescence from Cu-diffused $\mathrm{LiGaO}_{2}$ crystals, J. Lumin. 170 (2016) 17e23.

[12] J.-Y. Ma, F. Xu, M. Kamran, H.-Y. Zhao, C.-Z. Bi, B.-R. Zhao, X.-G. Qiu, Tem-perature dependent phonon modes and ionicity of $\mathrm{LiGaO}_{2}$ single crystal, Chin. Phys. B 17 (9) (2008) $3313 \mathrm{e} 3317$.

[13] M. Marezio, The crystal structure of $\mathrm{LiGaO}_{2}$, Acta Cryst. 18 (1965) 481e484.

[14] C. Chen, C.-A. Li, S.-H. Yu, M.M.C. Chou, Growth and characterization of b-LiGaO2 single crystal, J. Cryst. Growth 402 (2014) 325 e329.

[15] G. Dirksen, A.N.J.M. Hoffman, T.P. van de Bout, M.P.G. Laudy, G. Blasse, Luminescence spectra of pure and doped $\mathrm{GaBO}_{3}$ and $\mathrm{LiGaO}_{2}$, J. Mater. Chem. 1 (6) (1991) $1001 \mathrm{e} 1006$.

[16] N.W. Johnson, J.A. McLeod, A. Moewes, The electronic structure of lithium matagallate, J. Phys. Condens. Mat. 23 (2011) 445501.

[17] S. Tumenas, P. Mackonis, V. Karpus, R. Nedzinskas, L. Trinkler, B. Berzina, L. Chang, M.M.C. Chou, Optical properties of lithium gallium oxide, Appl. Surf.
Sci. (2016) (in press)

[18] A.V. Gaister, E.V. Zharikov, G.M. Kuz'micheva, Y.M. Papin, V.B. Rybakov, V.A. Smirnov, Octahedral $\mathrm{Cr}^{3 \mathrm{p}}$ sites in $\mathrm{LiGaO}_{2}$, Dokl. Phys. 45 (5) (2000) $172 \mathrm{e} 176$.

[19] M.L. Meylman, Luminescence spectra of chromium-doped $\mathrm{LiGaO}_{2}$ crystals as indicato of their phase heterogeneity, Opt. Mater. 28 (2006) $221 \mathrm{e} 224$.

[20] Y.J. Hsiao, T.-H. Fang, L.-W. Ji, Z.-W. Chiu, Novel photoluminescent properties of LiGaO2 nanoflakes, J. Alloys Compd. 509 (2011) 7684e7687.

[21] p.seiev M. Kato, K. Jarasiunas, A diffraction-based technique for determination of interband absorption coefficients in bulk $3 \mathrm{C}-, 4 \mathrm{H}-$ and $6 \mathrm{H}-\mathrm{SiC}$ crystals, J. Phys. D. Appl. Phys. 44 (2011) 365402.

[22] p.scaiev $\quad$ V. Gudelis, K. Jarasiunas, P.B. Klein, Fast and slow carrier recombination transients in highly excited $4 \mathrm{H}$ - and $3 \mathrm{C}-\mathrm{SiC}$ crystals at room temperature, J. Appl. Phys. 108 (2010) 023705.

[23] p. Scajev, J. Jurkevicius, J. Mickevicius, K. Jarasiunas, H. Kato, Features of free carrier and exciton recombination, diffusion, and photoluminescence in undoped and phosphorus-doped diamond layers, Diam. Relat. Mater. 57 (2015) 9 e16.

[24] D.J. Huntley, An explanation of the power-law decay of luminescence, J. Phys. Condens. Mat. 18 (2006) 1359e1365.

[25] B.K. Ridley, Quantum Processes in Semiconductors, Clarendon Press, Oxford, 1999.

[26] P.T. Landsberg, Trap-Auger recombination in silicon of low carrier densities, Appl. Phys. Lett. 50 (1987) 745.

[27] D.G. Thomas, J.J. Hopfield, W.M. Augustyniak, Kinetics of radiative recombination at randomly distributed donors and acceptors, Phys. Rev. 140 (1965) A202eA220.

[28] K. Vanheusden, C.H. Seager, W.L. Warren, D.R. Tallant, J. Voigt, Correlation between photoluminescence and oxygen vacancies in $\mathrm{ZnO}$ phosphors, Appl. Phys. Lett. 68 (1996) 403 e405.

[29] L. Trinkler, B. Berzina, Localised transitions in luminescence of AlN ceramics, Rad. Meas. 71 (2014) 232e236.

[30] J. Omachi, T. Suzuki, K. Kato, N. Naka, K. Yoshioka, M. Kuwata-Gonokami, Observation of excitonic N-body bound states: polyexcitons in diamond, Phys. Rev. Lett. 111 (2013), 026402e1-5.

[31] A. Hangleiter, R. Hacker, Enhancement of band-to-band Auger recombination by electron-hole correlations, Phys. Rev. Lett. 65 (1990) 215.

[32] H. Morimoto, Y. Hazama, K. Tanaka, N. Naka, Ultrahigh exciton diffusion in intrinsic diamond, Phys. Rev. B 92 (2015), 201202(R)-1-5. 